\title{
Anatomie clinique de l'articulation
}

\section{temporo-mandibulaire}

\section{RÉSUMÉ}

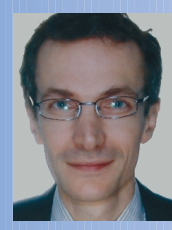

\section{Christian VACHER}

AP-HP,

Anatomie,

Faculté de médecine Diderot Paris VII et Service de chirurgie maxillo-faciale et stomatologie,

Hôpital Beaujon.
L'articulation temporo-mandibulaire présente des particularités fonctionnelles et cliniques. Si on étudie uniquement ses surfaces articulaires, c'est une articulation bi-condylaire particulièrement instable. Sa stabilité est renforcée par l'existence d'un disque articulaire qui sépare l'articulation en deux compartiments, supra et infra discal. Ses ligaments surtout présents à la face médiale de l'articulation augmentent sa stabilité. Les muscles moteurs de cette articulation sont tous innervés par le nerf mandibulaire. L'innervation sensitive dépend également du nerf mandibulaire ce qui explique le cercle vicieux qui s'installe dans les dysfonctionnements de cette articulation, la douleur entretenant le spasme des muscles masticateurs qui lui-même accentue la douleur. 


\section{Introduction}

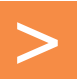

En anatomie, une articulation est un

compromis entre mobilité et stabilité. Plus une articulation est stable (comme la hanche) et moins elle est mobile. Plus une articulation est mobile (comme l'épaule) et moins elle est stable et peut se luxer. Les moyens de stabilité de l'articulation sont:

a. Les surfaces articulaires ;

\section{Description}

\section{Les surfaces articulaires[1]}

Au niveau de l'os temporal, le compartiment supérieur de l'articulation appartient à la partie squameuse du temporal (fig. 1). II présente d'avant en arrière le tubercule articulaire du temporal puis la fosse glénoïde, puis le tubercule post-glénoïde. Dans le fond de la fosse glénoïde apparaît la fissure pétro-tympanosquameuse qui fait communiquer l'oreille moyenne avec I'ATM. Certains croyaient que cette communication était l'explication des b. Les moyens d'union : disque articulaire et ligaments ;

c. Les muscles à la fois moyen d'union et de mobilité.

Le but de cet article est de présenter une anatomie clinique simple de l'articulation temporo-mandibulaire, à partir de 6 dessins d'anatomie.

signes otologiques observés dans les dysfonctions de l'ATM. Seule la partie de la fosse mandibulaire située en avant de la fissure pétrotympano-squameuse et le tubercule articulaire sont des surfaces articulaires. Le condyle mandibulaire est une saillie osseuse qui constitue le compartiment inférieur de l'articulation (fig. 2). Les deux surfaces articulaires sont convexes, I'ATM est une articulation bicondylaire et si I'on considère seulement les surfaces articulaires, elle est très instable.

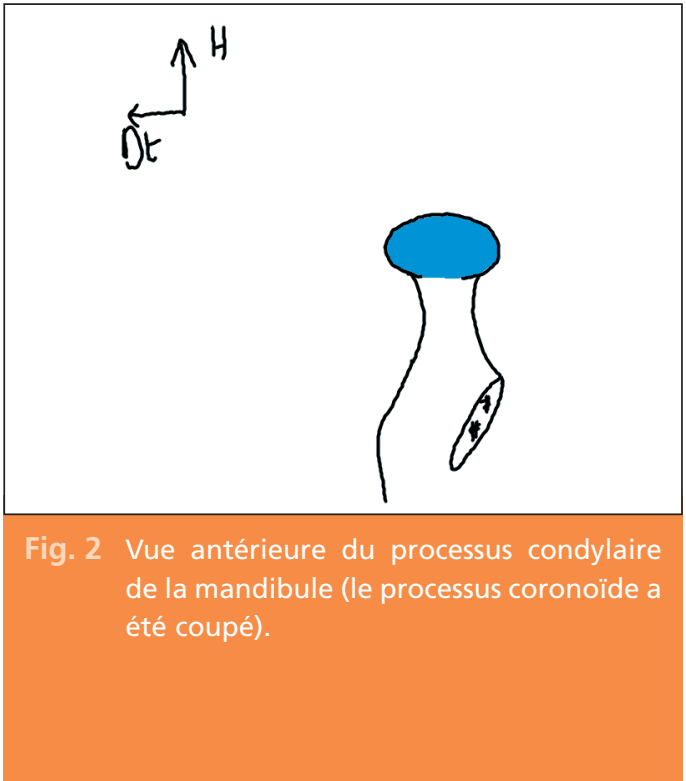




\section{Les moyens d'union[2]}

Pour augmenter la stabilité et assurer une meilleure coaptation des surfaces articulaires, il existe un disque articulaire qui est une lentille biconcave formée de tissu fibreux et conjonctif (fig. 3). II divise l'articulation en deux compartiment supra-discal (disco-temporal) et infra-discal (disco-mandibulaire). Ce disque présente trois parties :

- le 1/3 antérieur est situé en avant du condyle mandibulaire en occlusion, il est attaché à la capsule articulaire et au chef supérieur du muscle ptérygoïdien latéral ;

- le 1/3 moyen (partie la plus fine) est en regard du tubercule articulaire en occlusion ;

- le 1/3 postérieur est situé au-dessus du condyle en occlusion. Il se continue par la zone bi-laminaire. Cette structure est formée par une lame supérieure qui s'attache au tubercule postglénoïde, une lame inférieure qui s'attache au condyle mandibulaire et entre les deux un espace rétro discal riche en vaisseaux et en

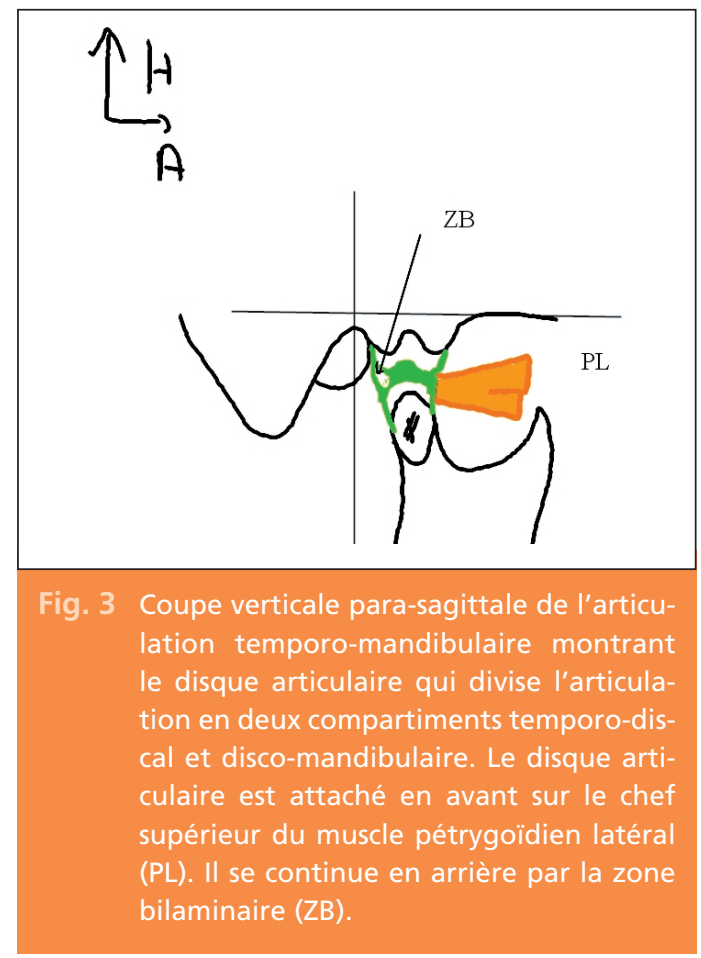

nerfs sensitifs. La présence de ces nerfs explique les douleurs articulaires lorsque le disque est en situation antérieure de manière prolongée.

La stabilité de l'articulation est également renforcée par des ligaments qui sont (à l'exception d'un frêle ligament latéral) présents à la face médiale de I'articulation (fig. 4). Le ligament sphéno-mandibulaire est un reliquat du cartilage de Meckel qui sert de guide à la croissance de la mandibule pendant la période embryonnaire et fœtale. Le ligament stylo-mandibulaire et un petit ligament ptérygo-mandibulaire complètent ces moyens d'union ligamentaires.

\section{Les muscles moteurs de l'ATM[3]}

Le muscle ptérygoïdien médial (fig. 4) s'insère dans la fosse interptérygoïdienne, se dirige obliquement en bas et en arrière et se termine à la face médiale de l'angle de la mandibule. Il

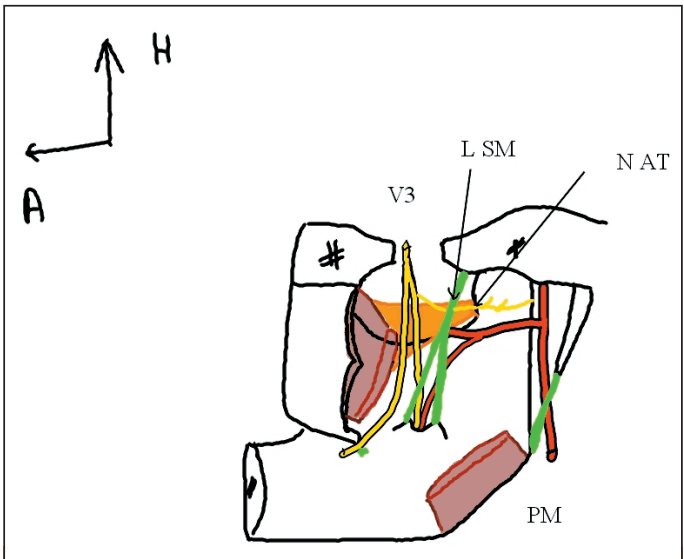

Fig. 4 Vue médiale de la région de l'articulation temporo-mandibulaire. Le muscle ptérygoïdien médial (PM) est tendu entre la fosse inter-ptérygoïdienne et la face médiale de l'angle mandibulaire. Le ligament sphéno-mandibulaire (L SM) se termine au niveau de la lingula mandibulaire. Le nerf mandibulaire (V3) reçoit le nerf auriculo-temporal (N AT). 
est élévateur, propulseur de la mandibule et diducteur.

Le muscle ptérygoïdien latéral (fig. 4) s'insère sur la face latérale de la lame latérale du processus ptérygoïde et se termine sur le col du conyle mandibulaire. II se prolonge par le disque articulaire. Il est propulseur de la mandibule lorsque les deux ptérygoïdien latéraux se contractent ensemble et diducteur quand un seul muscle se contracte.

Les autres muscles masticateurs sont visibles sur une vue latérale de la tête (fig. 5).

Le masséter s'insère sur le bord inférieur de l'arcade zygomatique et se termine sur la face latérale du ramus mandibulaire. II est élévateur de la mandibule. Le muscle temporal s'insère sur la fosse temporale et se termine en s'étalant sur le processus coronoïde de la mandibule. Il est élévateur et rétro-pulseur de la mandibule.

Il ne faut pas oublier l'existence des muscles abaisseurs de la mandibule qui sont le muscle digastrique et le muscle mylo-hyoïdien.

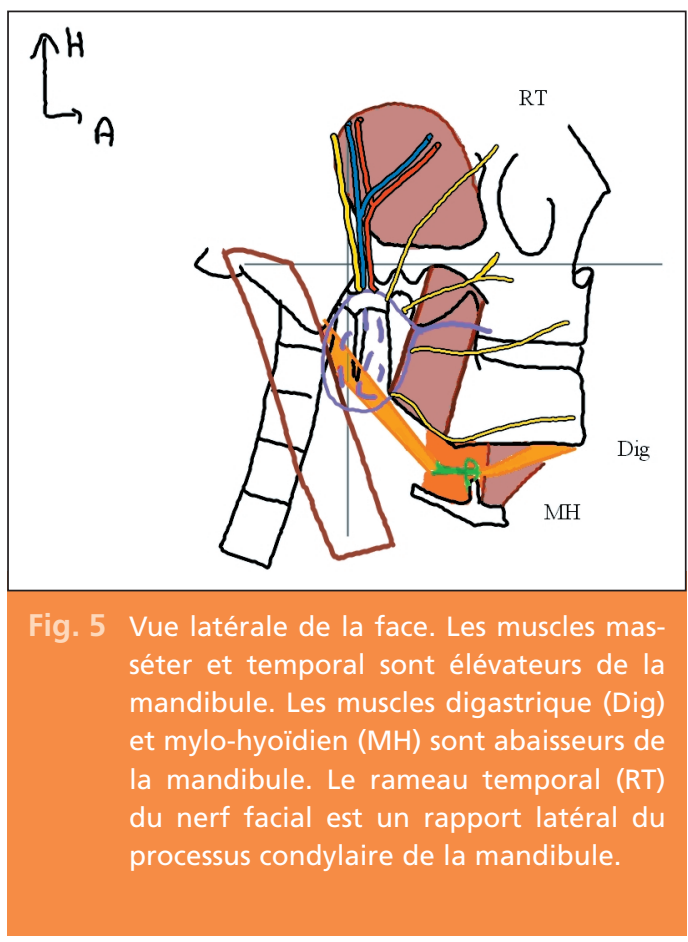

\section{Innervation de l'ATM[4]}

L'innervation sensitive dépend du nerf auriculotemporal, rameau sensitif du nerf mandibulaire qui participe également à l'innervation sensitive de la fosse temporale et de l'oreille externe. Ceci explique que les patients qui présentent un dysfonctionnement de l'ATM présentent également souvent des otalgies. Ce nerf passe à la face médiale du col du condyle mandibulaire pour rejoindre le nerf mandibulaire (fig. 4).

L'innervation motrice est sous la dépendance du nerf mandibulaire. Ce nerf donne après sa sortie par le foramen ovale (fig.6), des rameaux destinés au muscle temporal (nerfs temporaux profonds antérieur, moyen et postérieur), deux rameaux pour le muscle ptérygoïdien latéral (un pour chaque chef), et le nerf massétérique. II donne également un tronc commun pour les nerfs du ptérygoïdien médial, du tenseur du voile et du tenseur du tympan. On peut dès lors comprendre qu'en cas de spasme musculaire véhiculé par le nerf mandibulaire, il se produit non seulement un spasme

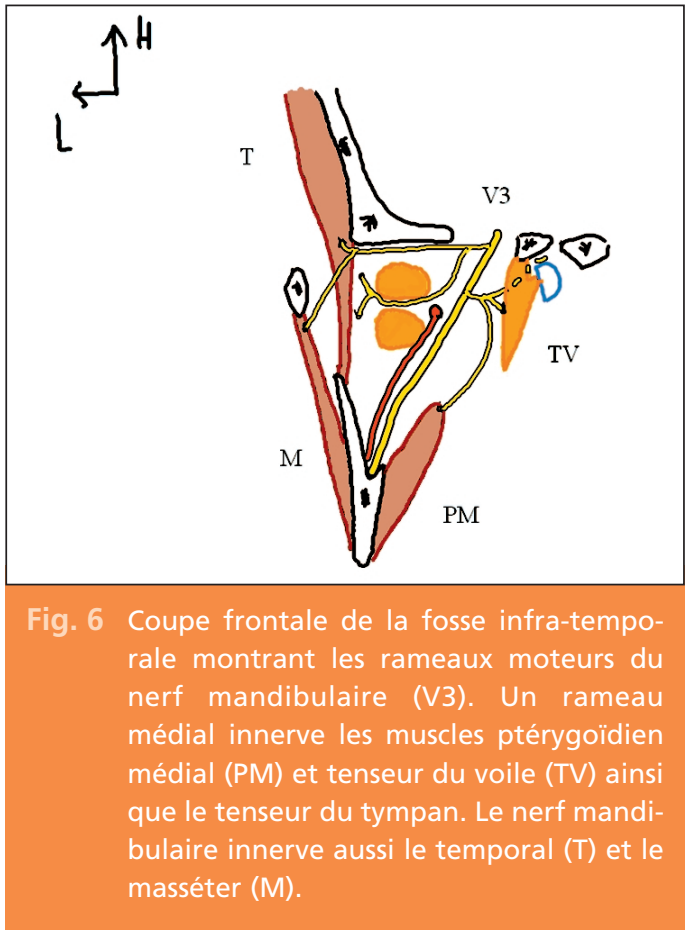


des muscles masticateurs, mais aussi un retentissement sur la trompe auditive (d'Eustache) par exemple une dysperméabilité tubaire, et sur l'oreille moyenne, ce qui explique les signes otologiques observés en cas de dysfonction de I'ATM. Ce spasme est entraîné soit par une hyperactivité de l'articulation soit par une anomalie portant sur l'occlusion dentaire.

\section{Conclusion}

L'anatomie de l'articulation temporo-mandibulaire explique assez bien les manifestations cliniques observées dans les dysfonctions des ATM. La douleur, qui peut être localisée à l'articulation elle-même par mise en tension de la zone bi-laminaire, peut également se présenter comme une otalgie car le nerf auriculo-temporal innerve l'oreille externe, ou comme une céphalée temporale car ce même nerf donne la sensibilité de la fosse temporale. La douleur peut être également musculaire en raison du spasme des muscles masticateurs innervés par le nerf mandibulaire. Les signes otologiques s'expliquent par l'innervation par le même nerf mandibulaire des muscles tenseur du voile que I'on appelait auparavant peristaphylin externe et tenseur du tympan.

\section{Bibliographie}

1. Georges $\mathrm{O}$. Tête et cou. Fascicule 1 Face et cou. Paris, G. Doin, 1961.

2. Grégoire et Oberlin. Précis d'anatomie. $11^{\mathrm{e}}$ édition.

Paris, Lavoisier,

2004.

3. Gaudy JF.

Anatomie clinique.

Paris, CDP, 2003.
4. Nortons NS.

Netter's head

and neck anatomy

for dentistry.

Philadelphia,

Saunders Elsevier, 2007.

\section{SUMMARY}

\section{Clinical anatomy of the temporomandibular joint}

Christian VACHER

$$
\begin{aligned}
& \text { Keywords } \\
& \text { - temporomandibular } \\
& \text { joint } \\
& \text { - anatomy } \\
& \text { - masticatory apparatus }
\end{aligned}
$$

The temporomandibular joint is a particular joint in a functional and clinical point of view. Considering its articular surfaces, it is a non stable bicondylar joint. Its stability is improved by the presence of an articular disc which divides the joint in two compartments, supra and infra discal. Its ligaments, located on the medial aspect of the joint, improved its stability. The motor muscles of the joint are all innervated by the mandibular nerve. The sensitive innervation is also mediated by the mandibular. This is the reason why in case of dysfunction of the joint, the pain improves the muscular spasm of the masticator muscles which improves the pain. 
Pour s'abonner, composer le :

tél. + 33 (0)1 $69181514-\mathrm{fax}+33(0) 169860678$

France et Union Européenne

version papier + version électronique $199 €$

Étudiant* : France et Union Européenne

(* sur présentation d'une attestation)

version papier + version électronique $119 €$

Reste du monde

version papier + version électronique $229 €$

Membre de I'AEOS

$179 €$

Prix au numéro

$65 €$

Frais d'envoi par avion

$49 €$

\section{NUMÉROS(*)}

(*) Offre valable jusqu'au 31/10/09

Réglé la somme de le

Chèque bancaire / postal $\mathrm{N}^{\circ}$

ou carte $\mathrm{N}^{\circ}$

(Partie à conserver)

(Partie à joindre à tout règlement)

$\square$ Je souhaite m'abonner pour

à la Revue AOS

$\square$ Je souhaite prolonger mon abonnement pour

à la Revue AOS

\section{MODE DE PAIEMENT :}

$\square$ Paiement effectué par chèque à l'ordre de : EDP Sciences - REVUE AOS

17 avenue du Hoggar - P.A. de Courtabœuf

B.P. 112 - 91944 Les Ulis Cedex A (France)

Tél. + 33 (0)1 69181514 - Fax + 33 (0)1 69860678

e-mail : abonnements@edpsciences.org

Paiement par carte bancaire : $\square$ Carte Visa

Master Card

$\mathrm{N}^{\circ}$

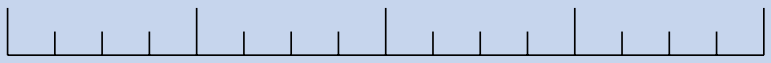

Noter ici les 3 derniers chiffres situés au dos de la carte

Date d'expiration $\lfloor|1|$

Signature obligatoire

\section{VOTRE ADRESSE :}

Vous êtes: $\square$ Chirurgien-Dentiste $\square$ Stomatologue $\square$ Orthodontiste $\square$ Autre: précisez

Nom :

Prénom :

Adresse :

Code Postal

Ville

Tél

Fax

E-mail 